\title{
FINITE DIMENSIONAL APPROXIMATIONS TO SOME FLOWS ON THE PROJECTIVE LIMIT SPACE OF SPHERES
}

\author{
HISAO NOMOTO
}

$\S 1$. Introduction. Let $\Omega$ be the projective limit space of a sequence of probability space $\Omega_{n}$ which is a certain subset of $(n-1)$-dimensional sphere with the usual uniform probability distribution on it. T. Hida [2], starting from a sequence of finite dimensional flows $\left\{T_{t}^{(n)}\right\}$ which are derived from some one-parameter subgroups of rotations of spheres, constructed a flow $\left\{T_{t}\right\}$ on $\Omega$ as the limit of them. Observing his method, the concept of consistency of flows $\left\{T_{t}^{(n)}\right\}$ which approximate $\left\{T_{t}\right\}$ seems to play an essential role in his work [2]. As will be made clear in the following sections, the concept of consistency is closely related to the projective limiting structure of our basic space $\Omega$. The purpose of this paper is to determine all the flows on $\Omega$ which can be approximated in the sense of [2] by finite dimensional flows.

In the first part of Section 2, some results in [3] which are needed for the following discussions are summarized, and later it is proved that a sequence of consistent flows determines a flow on $\Omega$. Further we prove that such finite dimensional flows derived from one-parameter subgroups of rotations will be characterized from the standpoint both of their forms and of their spectral sets. In Section 3, we shall consider the converse problem to construct a sequence of flows which approximate a given one on $\Omega$, and find a condition that these finite dimensional flows are one-parameter subgroups of rotations. Although it can be pointed out that $T_{t}^{(n)}$ converges to $T_{t}$ in the sense of weak topology introduced in the set of all automorphisms on $\Omega$, we shall not concern with further problems on this subject.

Finally, we would like to note that, in most places of our discussions, it is not necessary to avail ourselves of entire spheres if we consider set transformations instead of point transformations.

The author wishes to express his thanks to Professor T. Hida who has

Received March 31, 1966. 
encouraged him with kind discussions.

$\S 2$. Limit of flows on finite dimensional spheres. Let $S_{n}$ be $(n-1)$-sphere with radius $\sqrt{n}, \mathbf{B}\left(S_{n}\right)$ be the topological Borel field and $P_{n}$ be the uniform probabity measure on $S_{n}$. Let $x^{(n)}=\left(x_{1}^{(n)}, \ldots, x_{n}^{(n)}\right)$ be a point of $S_{n}$. Then $x^{(n)}$ can be expressed in the form

$$
\begin{aligned}
& x_{1}^{(n)}=\sqrt{n} \prod_{i=1}^{n-1} \sin \theta_{i}, \\
& x_{k}^{(n)}=\sqrt{n} \cos \theta_{k-1} \prod_{i=k}^{n-1} \sin \theta_{i}, 2 \leq k \leq n-1, \\
& x_{n}^{(n)}=\sqrt{n} \cos \theta_{n-1},
\end{aligned}
$$

where $0 \leq \theta_{1}<2 \pi, 0 \leq \theta_{i} \leq \pi(2 \leq i \leq n-1)$. Let $\Omega_{n}$ be an open subset of $S_{n}$ defined by

$$
\Omega_{n}=\left\{x^{(n)} ; x^{(n)} \in S_{n}, 0<\theta_{i}<\pi, i=2, \ldots, n-1\right\}
$$

and $\mathbf{B}_{n}$ be the family of Borel subsets of $\Omega_{n}$. We shall denote the restriction of $P_{n}$ to $\mathbf{B}_{n}$ by the same letter. Let $f_{m, n}(m<n)$ be a point transformation from $\Omega_{n}$ onto $\Omega_{m}$ such that if the polar coordinates of $x^{(n)} \in \Omega_{n}$ are $\theta_{1}, \ldots, \theta_{n-1}$, then those of $x^{(m)}=f_{m, n}\left(x^{(n)}\right)$ are $\theta_{1}, \ldots, \theta_{m-1}$. In other words,

$$
x_{k}^{(m)}=\frac{\sqrt{m}}{\left[x_{1}^{(n) 2}+\cdots+x_{m}^{(n) 2}\right]^{1 / 2}} x_{k}^{(n)}, k=1, \ldots, m .
$$

This $f_{m, n}$ determines a homomorphic mapping from the measure space $\left(\Omega_{n}, \mathbf{B}_{n}, P_{n}\right)$ to $\left(\Omega_{m}, \mathbf{B}_{m}, P_{m}\right)$. Moreover, the system $\left[\left(\Omega_{n}, \mathbf{B}_{n}, P_{n}\right) ; f_{m, n}\right]$ is a sequentially maximal topological stochastic family in the sense of $\mathrm{S}$. Bochner, so that we can form a probability space $(\Omega, \mathbf{B}, P)$ which is the projective limit of this system ([1], [3]). By definition of projective limit, we have

$$
\begin{aligned}
& f_{l, n}=f_{l, m} \circ f_{m, n}, \quad l<m<n, \\
& f_{m}=f_{m, n} f_{n},
\end{aligned}
$$

where $f_{n}$ denotes the projection from $\Omega$ onto $\Omega_{n}$.

Let $M^{\prime}$ be a subset of the set $M=\{2,3, \ldots\}$. Assume that we are given a point transformation $T^{\left(n^{\prime}\right)}$ on $S_{n^{\prime}}$ ( or $\Omega_{n^{\prime}}$ ), for each $n^{\prime} \in M^{\prime}$. Then we shall call the system $\left\{T^{\left(n^{\prime}\right)} ; n^{\prime} \in M^{\prime}\right\}$ to be consistent if, for any $m^{\prime}, n^{\prime}$ in $M^{\prime}$, either the relation

$$
T^{\left(m^{\prime}\right)} \circ f_{m^{\prime}, n^{\prime}}=f_{m^{\prime}, n^{\prime}} \circ T^{\left(n^{\prime}\right)} \text { on } \Omega_{n^{\prime}},
$$


or the relation

$$
T^{\left(m^{\prime}\right)} \circ f_{m^{\prime}, n^{\prime}}=f_{m^{\prime}, n^{\prime}} \circ T^{\left(n^{\prime}\right)} \text { on } \Omega_{n^{\prime}}(\bmod 0),
$$

holds according as each $T^{(\cdot)}$ is an automorphism or an automorphism $(\bmod 0)$ on $\Omega_{n^{\prime}}$.

Let $M^{\prime}=\left\{n_{p} ; p \geq 1\right\}$ be a cofinal subset of the above set $M$ and let $(\widetilde{\Omega}, \widetilde{\mathbf{B}}, \widetilde{P})$ be a projective limit probability space which is determined by the subsystem $\left[\left(\Omega_{n^{\prime}}, \mathbf{B}_{n^{\prime}}, P_{n^{\prime}}\right) ; f_{m^{\prime}, n^{\prime}} ; m^{\prime}, n^{\prime} \in M^{\prime}\right]$. Then, since $\widetilde{\Omega}$ is a closed subset of the weak product $\prod_{n^{\prime} \in M^{\prime}} \Omega_{n^{\prime}}$, it is a Borel set of $S_{\infty}=\prod_{n^{\prime} \in M^{\prime}} S_{n}$. (weak product). Therefore, we can define a probability measure $P_{\infty}$ on $\mathbf{B}_{\infty}$ (topoligical Borel field of $\left.S_{\infty}\right)$ so as $P_{\infty}(A)=\widetilde{P}(A \cap \widetilde{\Omega})$, for each $A \in \mathbf{B}_{\infty}$. Now, let $p_{n^{\prime}}$ be the projection from $S_{\infty}$ onto $S_{n^{\prime}}$. Then we have the following proposition.

Proposition 2.1. Let $\left\{T^{\left(n^{\prime}\right)} ; n^{\prime} \in M^{\prime}\right\}$ be a consistent system of automorphisms on $S_{n^{\prime}}$. Then the mapping defined by

$$
T: S_{\infty} \ni x \rightarrow T x=\left(T^{\left(n^{\prime}\right)} \circ p_{n^{\prime}}(x) ; n^{\prime} \in M^{\prime}\right)
$$

gives an automorphism on $S_{\infty}$, and $T$ induces an automorphism (mod 0$)$ on $\Omega$. If $T^{\left(n^{\prime}\right)}$ is an automorphism ( $\left.\bmod 0\right)$ on $\Omega_{n^{\prime}}$, then the transformation defined by

$$
T: \widetilde{\Omega} \ni x \rightarrow T x=\left(T^{\left(n^{\prime}\right)} \circ f_{n^{\prime}}(x) ; n^{\prime} \in M^{\prime}\right)
$$

determines an automorphism $(\bmod 0)$ on $\widetilde{\Omega}$.

Proof. We need to prove only the first part of the proposition. By definition of $T$, it is a one-to-one mapping from $S_{\infty}$ onto $S_{\infty}$. Since each $T^{\left(n^{\prime}\right)}$ is an automorphism on $S_{n^{\prime}}$, there exists a subset $\Omega_{n^{\prime}}^{\gamma}$ of $\Omega_{n}$. such that $T^{\left(n^{\prime}\right)}$ is an automorphism on $\Omega_{n^{\prime}}^{\circ}$. Now, put $\Omega^{\circ}=\bigcap_{n^{\prime} \in M^{\prime}} f_{n^{\prime}}^{-1}\left(\Omega_{n^{\prime}}^{\circ}\right)$. If $x \in \Omega^{\circ}$, then, in view of (2.2) and (2.3)', we have

$$
T^{\left(m^{\prime}\right)} \circ f_{m^{\prime}}(x)=T^{\left(m^{\prime}\right)} \circ f_{m^{\prime}, n^{\prime}} \circ f_{n^{\prime}}(x)=f_{m, n^{\prime}} \circ T^{\left(n^{\prime}\right)} \circ f_{n^{\prime}}(x) \text {, for } m^{\prime}<n^{\prime} .
$$

This shows that the point $\left(T^{\left(n^{\prime}\right)} \circ f_{n^{\prime}}(x) ; n^{\prime} \in M^{\prime}\right)$ is in $\Omega^{\circ}$, so that $T$ maps $\Omega^{\circ}$ into $\Omega^{\circ}$. Moreover, it is easy to show that $T$ is a one to one onto mapping. Let $A$ be a Borel set of $\Omega^{\prime \prime} \cap f_{n^{\prime}}^{-1}\left(\mathbf{B}_{n}\right)$, then (2.3)' implies $T A=f_{n^{\prime}}^{-1} \circ T^{\left(n^{\prime}\right)} \circ f_{n^{\prime}}(A)$, so that we have

$$
\widetilde{P}(T A)=\widetilde{P}\left(f_{n^{\prime}}^{-1} \circ T^{\left(n^{\prime}\right)} \circ f_{n^{\prime}}(A)\right)=P_{n^{\prime}}\left(T^{\left(n^{\prime}\right)} \circ f_{n^{\prime}}(A)\right)=P_{n^{\prime}}\left(f_{n^{\prime}}(A)\right)=\widetilde{P}(A) .
$$

$T$ is therefore, measure preserving on $\Omega^{\circ}$, since $\underset{n^{\prime} \in M^{\prime}}{\bigvee} f_{n^{\prime}}^{-1}\left(\mathbf{B}_{n^{\prime}}\right)=\widetilde{\mathbf{B}} \cap \Omega^{\circ}$. By the 
same arguments as above, $T^{-1}$ is also measure preserving. Thus $T$ is an automorphism on $\dot{\Omega}^{\circ}$, therefore it is automorphic on $S_{\infty}$. To conclude the proof, it is enough to show that $\Omega^{\circ}$ is isomorphic $(\bmod 0)$ to $\Omega$. This fact is, however, a direct consequence of sequential maximal property of $\left\{f_{m, n}\right\}$.

By using the same notations as above, we have the following theorem.

THEOREM 2.1. Let $\left\{T_{t}^{\left(n^{\prime}\right)}\right\}$ be a measurable flow on $S_{n^{\prime}}$ and let $\left\{T_{t}^{\left(n^{\prime}\right)} ; n^{\prime} \in M^{\prime}\right\}$. be consistent for each $t$. Then, there exists a measurable flow $\left\{T_{t}\right\}$ on $S_{\infty}$ such that

$$
p_{n} \circ T_{t}=T_{t}^{\left(n^{\prime}\right)} \circ p_{n} ; \text { on } S_{\infty} \text {. }
$$

Proof. Define $T_{t}$ by (2.4), then it is an automorphism on $S_{\infty}$. It is easy to see that the group property $T_{s} T_{t}=T_{s+t}$ holds. To prove the theorem, it is enough to show that $\left\{T_{t}\right\}$ is measurable. Let $f(x)$ be a tame function on $S_{\infty}$ such that $f(x)=\prod_{n^{\prime} \in N^{\prime}} \varphi_{n^{\prime}} \circ p_{n^{\prime}}(x)$, where $N^{\prime}$ is a finite subset of $M^{\prime}$, and every $\varphi_{n^{\prime}}$ is a continuous function on $S_{n^{\prime}}$. Then, $f\left(T_{t} x\right)=\prod_{n^{\prime} \in N^{\prime}} \varphi_{n^{\prime}} \circ p_{n^{\prime}}\left(T_{t} x\right)=$ $\prod_{n^{\prime} \in N^{\prime}} \varphi_{n^{\prime}}\left(T_{t}^{\left(n^{\prime}\right)} \circ p_{n}(x)\right)$ is $(t, x)$ measurable, since $\left\langle T_{t}^{\left(n^{\prime}\right)}\right\}$ is measurable. It is known that the set of all continuous tame functions on $S_{\infty}$ is dense in the set of all contiuous functions on $S_{\infty}$ in the sense of uniform convergence, so that $T_{t} x$ is $(t, x)$ measurable. This proves the theorem.

Now we shall consider a measurable flow which is derived from a oneparameter subgroup of $S O(n)$. To discuss the consistency condition $(2.3)^{\prime}$ we prepare lemmas.

Lemma 2.1. Let $T^{(m)}$ and $T^{(n)}$ be automorphisms on measure spaces $S_{m}$ and $S_{n}$ which are derived from rotations of spheres $S_{m}$ and $S_{n}$ respectively. Provided that $n<2 m$, if they form consistent system, then $T^{(n)}$ is expressed in the following form:

$$
T^{(n)}=\left[\begin{array}{c:c}
T^{(m)} & 0 \\
\hdashline 0 & T^{(n-m)}
\end{array}\right]
$$

Conversly, if $T^{(n)}$ is of the above form, then $T^{(m)}$ and $T^{(n)}$ are consistent.

Proof. $1^{\circ}$. Let $x^{(n)}=\left(x_{1}^{(n)}, \ldots, x_{m}^{(n)}, 0, \ldots, 0\right)$ be a point of $\Omega_{n}$ such that $y^{(n)}=T^{(n)} x^{(n)} \in \Omega_{n}$ and that $(2.3)^{\prime}$ holds. Putting $T^{(n)}=\left(a_{i j}\right)_{i, j=1, \ldots, n}, \widetilde{T}^{(m)}=$ $\left(a_{i j}\right)_{i, j=1, \ldots, m}$, and by using $(2.1)$, the relation $(2.3)^{\prime}$ can be written in the form 


$$
\frac{\sqrt{m}}{\left[x_{1}^{(n) 2}+\cdots+x_{m}^{(n) 2}\right]^{1 / 2}} T^{(m)}\left(\begin{array}{c}
x_{1}^{(n)} \\
\cdot \\
\cdot \\
\cdot \\
x_{m}^{(n)}
\end{array}\right)=\frac{\sqrt{m}}{\left[y_{1}^{(n) 2}+\cdots+y_{m}^{(n)}\right]^{1 / 2}} \widetilde{T}^{(m)}\left(\begin{array}{c}
x_{1}^{(n)} \\
\cdot \\
\cdot \\
\cdot \\
x_{m}^{(n)}
\end{array}\right)
$$

so that we have

$$
\left.T^{(m)-1} \widetilde{T}^{(m)} \mid \begin{array}{c}
x_{1}^{(n)} \\
\cdot \\
\cdot \\
\cdot \\
x_{m}^{(n)}
\end{array}\right)=\rho\left(\begin{array}{c}
x_{1}^{(n)} \\
\cdot \\
\cdot \\
x_{m}^{(n)}
\end{array}\right)
$$

where $\rho=\rho\left(x_{1}^{(n)}, \ldots, x_{m}^{(n)}\right)=\left[y_{1}^{(n) 2}+\cdots+y_{m}^{(n) 2}\right]^{1 / 2}\left[x_{1}^{(n) 2}+\cdots+x_{m}^{(n) 2}\right]^{-1 / 2}$. This shows that $\rho$ is an eigenvalue of $T^{(m)-1} \tilde{T}^{(m)}$ and that $\left(x_{1}^{(n)}, \ldots, x_{m}^{(n)}\right)$ is an eigenvector corresponding to $\rho$. Since sufficiently many points of $\Omega_{n}$ satisfy this relation, $\rho$ should be a constant such that $0<\rho \leq 1$, and we have $\widetilde{T}^{(m)}=\rho T^{(m)}$. Here note that $T^{(n)}$ is expressed in the following form

$$
T^{(n)}=\left[\begin{array}{c:c}
\rho T^{(m)} & \sigma^{\prime} B \\
\hdashline \sigma A & \rho^{\prime} C
\end{array}\right]
$$

where $A, B$ and $C$ are some matrices and $\rho^{\prime}= \pm \rho, \sigma, \sigma^{\prime}= \pm \sqrt{1-\rho^{2}}$.

$2^{\circ}$. Now assume that $n<2 m$. If $\rho<1$, then, since both $T^{(m)}$ and $T^{(n)}$ are orthogonal matrices, the column vectors of $\sigma A$ span $m$-dimensional subspace of $R^{n-m}$. But this is impossible since $n-m<m$, so that $\rho=1$.

LEMma 2.2. Let $\left\{T_{t}^{(m)}\right\}$ and $\left\{T_{t}^{(n)}\right\}$ be flows on $S_{m}$ and $S_{n}$ which are derived from one-parameter subgroups of $S O(m), S O(n)$ respectively. When $n \leq 2 m$, they form consistent system if and only if

$$
T_{t}^{(n)}=\left[\begin{array}{c:c}
T_{t}^{(m)} & 0 \\
\hdashline 0 & T_{t}^{(i n-m)}
\end{array}\right]
$$

Proof . $1^{\circ}$. If $n<2 m$, then $(2.8)$ is a consequence of Lemma 2.1. $2^{\circ}$. Now assume $n=2 \mathrm{~m}$. In the course of the proof of Lemma 2.1 we have shown that $T_{t}^{(n)}$ is of the form

$$
T_{t}^{(n)}=\left[\begin{array}{c:c}
\rho_{t} T_{t}^{(m)} & \sigma_{t}^{\prime} B_{t} \\
\hdashline \sigma_{t} A_{t} & \rho_{t}^{\prime} C_{t}^{\prime}
\end{array}\right]
$$

First, we note that the group property $T_{s}^{(\cdot)} T_{t}^{(.)}=T_{s+t}^{(.)}$implies

$$
\rho_{0}=1, \quad \rho_{t}=\rho_{-t},
$$


and that $\rho_{t}$ is continuous in $t$.

If $\rho_{t}<1$ for all $t \neq 0$, then it follows that $A_{t}, B_{t}$, and $C_{t}$ are all $m \times m$ orthogonal matrices. In view of the form of $T_{t}^{(\cdot)}$ and the group property, we have the following relation, by comparing left upper $m \times m$ matrices of $T_{t+s}^{(n)}$ and $T_{s}^{(m)} T_{t}^{(m)}$,

$$
\left(\rho_{s+t}-\rho_{s} \rho_{t}\right) T_{s+t}^{(m)}=\sigma_{t}^{\prime} \sigma_{s} B_{t} A_{s} .
$$

Therefore, by cnsidering the determinants of both sides, it follows that

$$
\left(\rho_{s+t}-\rho_{s} \rho_{t}\right)^{m}= \pm\left(\sigma_{t}^{\prime} \sigma_{s}\right)^{m}
$$

hence,

$$
\rho_{s+t}-\rho_{s} \rho_{t}= \pm \sigma_{t}^{\prime} \sigma_{s}= \pm \sqrt{1-\rho_{s}^{2}} \sqrt{1-\rho_{t}^{2}}
$$

Since $\rho_{t}$ is continuous in $t$, the left hand side of the above expression has constant sign, so that it should be either

Case 1.

$$
\rho_{s+t}-\rho_{s} \rho_{t}=\sqrt{1-\rho_{s}^{2}} \sqrt{1-\rho_{t}^{2}} \text { for all } s, t \neq 0,
$$

or

Case 2. $\quad \rho_{s+t}-\rho_{s} \rho_{t}=-\sqrt{1-\rho_{s}^{2}} \sqrt{1-\rho_{t}^{2}}$ for all $s, t \neq 0$.

In the case 1 , if we set $s=t$, then $\rho_{2 t}-\rho_{t}^{2}=1-\rho_{t}^{2}$, that is $\rho_{2}=1$ which is a contradiction. In the case 2 , if we let $s$ tend to $-t$, then the continuity of $\rho_{t}$ and (2.9) again imply a contradiction $1-\rho_{t}^{2}=-\left(1-\rho_{t}^{2}\right)$. Therefore, we have proved that $\rho_{t}=1$ for some $t \neq 0$. Observing the above arguments, we can restrict the range of variables $s$ and $t$ within any symmetric region around the origin which is bounded away from it. Hence it is easily seen that $\rho_{t}=1$ for all $t$. This proves the necessity of the theorem. Sufficiency is obvious.

Let $\left\{T_{t}^{(n)}\right\}$ be a one-parameter subgroup of $S O(n)$. Then, it is well known that $T_{t}^{(n)}$ can be expressed as the exponential form

$$
T_{t}^{(n)}=\exp (t X), X+X^{t}=0(X: \text { real-skew symmetric matrix }) .
$$

Let $\left\{ \pm i \lambda_{1}, \ldots, \pm i \lambda_{r}, 0, \ldots, 0\right\}\left(\lambda_{k} \neq 0\right)$ be the spectral set of $X$, then there exists an orthogonal matrix $T$ such that 
(2.10)

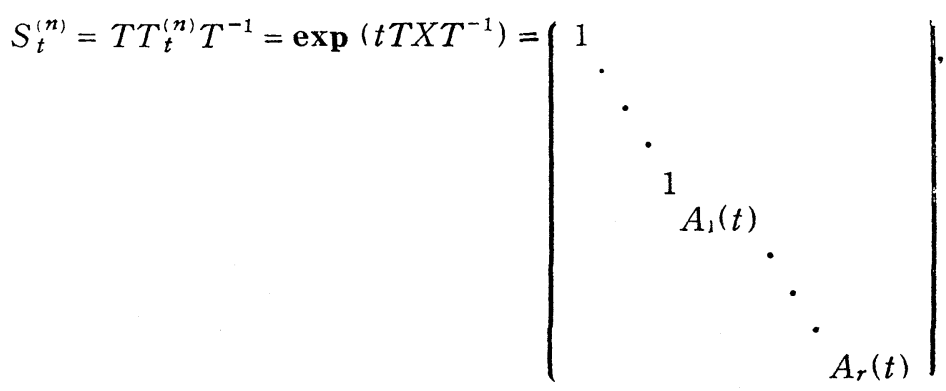

where $A_{k}(t)=\left[\begin{array}{lr}\cos \lambda_{k} t & -\sin \lambda_{k} t \\ \sin \lambda_{k} t & \cos \lambda_{k} t\end{array}\right]$.

This means that the flow $\left\{T_{t}^{(n)}\right\}$ is isomorphic to a flow of the above form. We shall say that the flow $\left\{S_{t}^{(n)}\right\}$ is canonical.

Let $M^{\prime}=\left\{n_{p} ; p \geq 1\right\}$ be a cofinal subset of $M=\{2,3, \ldots\}$ such that a condition

$$
2 n_{p} \geq n_{p+1}, \quad p \geq 1
$$

is satisfied and assume that we are given a one-parameter subgroup $\left\{T_{t}^{\left(n^{\prime}\right)}\right\}$ of $S O\left(n^{\prime}\right)$ for each $n^{\prime} \in M^{\prime}$. Then, we have the following theorem.

THEOREM 2.2. If the system of flows $\left\{T_{t}^{\left(\eta^{\prime}\right)}\right\}$ is consistent for each $t$, then the flow $\left\{T_{t}\right\}$ on $S_{\infty}$ which is determined by $\left\{T_{t}^{\left(n^{\prime}\right)}\right\}\left(n^{\prime} \in M^{\prime}\right)$ is isomorphic to a flow that is determined by a consistent system of "canonical" flows.

Proof. On account of Lemma 2.2, $T_{t}^{\left(n_{\mu}\right)}$ has a form

$$
T_{t}^{\left(\boldsymbol{n}_{p)}\right)}=\left(\begin{array}{lll}
\tilde{T}_{t}^{(1)} & & \\
& \cdot & \\
& \cdot & \\
& & \tilde{T}_{t}^{(p)}
\end{array}\right) \text {, where } \tilde{T}_{t}^{(1)}=T_{t}^{\left(\boldsymbol{n}_{1}\right)} .
$$

By the same arguments as in $(2.10)$, we can find $T^{\left(n_{p}\right)} \in O\left(n_{p}\right)$ such that

$$
T^{\left(n_{p}\right)}=\left|\begin{array}{lll}
\tilde{T}^{(1)} & & \\
& \cdot & \\
& \cdot & \\
& & \widetilde{T}^{(p)}
\end{array}\right|
$$

and 


$$
S_{t}^{\left(n_{p}\right)}=T^{(n p)} \cdot T_{t}^{\left(n_{p}\right)} \cdot T^{\left(n_{p}\right)-1}=\left(\begin{array}{lll}
\widetilde{S}_{t}^{(1)} & & \\
& \cdot & \\
& \cdot & \\
& & \widetilde{S}_{t}^{(p)}
\end{array}\right)
$$

where each $\left\{\widetilde{S}_{t}^{(k)}\right\}$ is canonical. Since $\left\{S_{t}^{\left(n_{p}\right)}\right\}$ is consistent, it determines a flow $\left\{S_{t}\right\}$ on $S_{\infty}$ and also $\left\{T^{\left(n_{p}\right)}\right\}$ gives an automorphism $T$ on $S_{\infty}$. Then it is obvious that $S_{t}=T \cdot T_{t} \cdot T^{-1}$. This concludes the proof.

Let $\Lambda_{p}=\left\{\lambda_{1}^{(p)}, \ldots, \lambda_{n_{p}-n_{p-1}}^{(p)}\right\}$ be the spectral set of $\left\{\widetilde{T}_{t}^{(p)}\right\}$ and $\Lambda$ be a sequence of numbers which are ordered in such a way: $\lambda_{1}^{(1)}, \ldots, \lambda_{n_{1}}^{(1)}, \lambda_{1}^{(2)}, \ldots$ For short, we shall call $\Lambda$ the spectral set of $\left\{T_{t}\right\}$.

Now, concerning Theorem 2.2, there is an alternative interpretation for consistent canonical flows even in case the condition $(*)$ is false. Assume that every fiow $\left\{T_{t}^{\left(n_{p}\right)}\right\}$ is given in the form (2.11). Then everything is the same as in Theorem 2.2. On the other hand, as we have seen in (2.10), each $\left\{T_{t}^{\left(n_{p}\right)}\right\}$ is uniquely determined by its spectral set up to metrical equivalence by orthogonal transformations. Therefore we have following proposition.

Proposition 2.2. Let $M^{\prime}=\left\{2 n_{p} ; p \geq 1\right\}$ be any cofinal subset of $M=\{2,3, \ldots\}$, and let $\Lambda=\left\{\lambda_{k} ; k \geq 1\right\}$ be a sequence of real numbers. Then, there exists a flow $\left\{T_{t}\right\}$ on $S_{\infty}=\prod_{n^{\prime} \in M^{\prime}} S_{n^{\prime}}$ with $A$ as its spectral set. This flow is uniquely determined by $A$ up to metrical equivalence, if we confine the consistent sequence which approximates $\left\{T_{t}\right\}$ to canonical ones.

Proposition 2.3. Let $\left\{T_{t}\right\}$ be a flow with spectral set $A$. Then $\left\{T_{t}\right\}$ is periodic with period $2 \pi$ if and only if $\Lambda$ is constituted by integers.

At this stage, we wish to mention a meaning of spectral set $\Lambda$ in our sense. In [2], Hida first constructed a flow $\left\{T_{t}\right\}$ with spectral set $\{0,1,2, \ldots\}$ when $M^{\prime}=\{3,5,7, \ldots\}$, which is closely related with periodical Brownian motion as was studied there. Following to him, let us consider the case where $M^{\prime}=$ $\{2,4,6, \ldots\}$ and $\Lambda=\left\{\lambda_{k} ; k \geq 1\right\},\left\{\lambda_{k}\right\}$ being an arbitrary sequence of reals. Denoting by $\left\{T_{t}\right\}$ the flow with $\Lambda$ as its spectral set, and we can assume that $T_{t}$ is constructed by canonical flows such that

$$
\tilde{T}_{t}^{(p)}=\left[\begin{array}{cc}
\cos \lambda_{p} t & -\sin \lambda_{p} t \\
\sin \lambda_{p} t & \cos \lambda_{p} t
\end{array}\right]
$$

(cf. (2.11)). It is known that $\lim _{n \rightarrow \infty} x_{k}^{(2 n)}=\lim _{n \rightarrow \infty}\left[k\right.$-th coordinate of $\left.f_{2 n}(x)\right]=\xi_{k}(x)$ 
exists (a.e. on $S_{\infty}$ ) and that $\left\{\xi_{k}\right\}$ is an independent Gaussian system such that each $\xi_{k}$.has mean 0 and variance 1 ([3, p. 302]). Therefore we have

$$
\begin{aligned}
& \xi_{2 k-1}\left(T_{t} x\right)=\xi_{2 k-1}(x) \cos \lambda_{k} t-\xi_{2 k}(x) \sin \lambda_{k} t, \quad\left(\text { a e. on } S_{\infty}\right) \\
& \xi_{2 k}\left(T_{t} x\right)=\xi_{2 k-1}(x) \sin \lambda_{k} t+\xi_{2 k}(x) \cos \lambda_{k} t,
\end{aligned}
$$

for each $t$. Further we have

$$
\xi_{a}(x)=\sum_{k \geq 1} a_{k} \xi_{k}(x)
$$

is a Gaussian random variable if $a=\left(a_{1}, a_{2}, \ldots\right)$ is in $\ell^{2}$. Since the correspondence $\sigma: \ell^{2} \ni a \rightarrow \xi_{a}$ in (2.15) is linear and isometric from $\ell^{2}$ to $\mathbf{L}^{2}\left(S_{\infty}\right)\left(\left[3\right.\right.$, p. 302]), we can say that the unitary group $U_{t}: f(x) \rightarrow f\left(T_{t} x\right)$ has discreate spectrum $\left\{ \pm \lambda_{k}\right\}$ on a closed linear manifold $\sigma\left(\ell^{2}\right)$ of $\mathbf{L}^{2}\left(S_{\infty}\right)$.

§ 3. Approximation to the flow on $(\Omega, \mathbf{B}, P)$. Let $\zeta_{n^{\prime}}$ be a partition of $\Omega$ derived from the projection $f_{n^{\prime}}: \Omega \rightarrow \Omega_{n^{\prime}}$, where $n^{\prime}$ is in $M^{\prime}$, the set presented before. Similarly let $\eta_{n}$, a partition of $S_{\infty}$, be defined by $p_{n^{\prime}}$. Here we introduce the following conditions:

$$
\zeta_{n^{\prime}} \uparrow \varepsilon, \eta_{n^{\prime}} \uparrow \delta \quad\left(n^{\prime} \uparrow \infty\right) .
$$

In other words, $\zeta_{n^{\prime}}\left(\eta_{n^{\prime}}\right)$ is a refinement of $\zeta_{m^{\prime}}\left(\eta_{m^{\prime}}\right)$ for $m^{\prime}<n^{\prime}$ and $\zeta_{n^{\prime}}$ tends to the pointwise partiton $\varepsilon(\delta)$ of $\Omega\left(S_{\infty}\right)$ as $n^{\prime} \uparrow \infty$ in the sense of the usual order of partitions. Let us consider an automorphism and an automorphism $(\bmod 0)$ $T$ on $\Omega . \quad T$ is said to be consistent with $\left\{\zeta_{n^{\prime}}\right\}$ either

$$
T \zeta_{n^{\prime}}=\zeta_{n^{\prime}}
$$

or

$$
T \zeta_{n^{\prime}}=\zeta_{n^{\prime}} \quad\left(\bmod 0 P_{\zeta_{n^{\prime}}}\right)
$$

holds, where $P_{\zeta_{n^{\prime}}}$ is the factor measure on the factor space $\Omega / \zeta_{n^{\prime}}$, which is isomorphic to $\Omega_{n^{\prime}}$. Similarly, we call an automorphism $S$ on $S_{\infty}$, is consistent with $\left\{\zeta_{n}\right\}$ if

$$
S \eta_{n^{\prime}}=\eta_{n^{\prime}} \text { and } S \zeta_{n^{\prime}}=\zeta_{n^{\prime}} \quad\left(\bmod 0 P_{\zeta_{n^{\prime}}}\right)
$$

In view of $(3.1)((3.2))$ or $(3.3)$, there exists a well defined point transformation on $\Omega$ or $S_{\infty}$ such that

$$
T^{\left(n^{\prime}\right)}=f_{n^{\prime}} T f_{n^{\prime}}^{-1}
$$


or

$$
S^{\left(n^{\prime}\right)}=p_{n^{\prime}} T p_{n^{\prime}}^{-1}
$$

We can now state a proposition.

Proposition 3.1. Suppose that the condition $(A)$ is satisfied. If $T(S)$ is an automorphism or automorphism $(\bmod 0)$ on $\Omega\left(S_{\infty}\right)$ which is consistent with $\left\{\zeta_{n^{\prime}}\right\}$, then $T^{\left(n^{\prime}\right)}\left(S^{\left(n^{\prime}\right)}\right)$ is an automorphism or automorphism $(\bmod 0)$ on $\Omega\left(S_{\infty}\right)$. Moreover $\left\{T^{\left(n^{\prime}\right)}\right\}\left(\left\{S^{\left(n^{\prime}\right)}\right\}\right)$ is consistent and determinines the given $T(S)$.

Proof. Let $\Omega^{\circ}$ be a subset of $\Omega$ on which $T$ is automorphic. It is easy to show that $T^{\left(n^{\prime}\right)}$ is automorphic on $\Omega_{n^{\prime}}^{\circ}=f_{n^{\prime}}\left(\Omega^{\circ}\right)$. By using (2.2) and (2.3), we have

$$
\begin{aligned}
T^{\left(m^{\prime}\right)} \cdot f_{m^{\prime}, n^{\prime}}\left(x^{\left(n^{\prime}\right)}\right)= & T^{\left(m^{\prime}\right)} \cdot f_{m^{\prime}, n^{\prime}} \cdot f_{n^{\prime}}(x)=T^{\left(m^{\prime}\right)} \cdot f_{m^{\prime}}(x)=f_{m^{\prime}}(T x) \\
= & f_{m, n^{\prime}} \cdot f_{n^{\prime}}(T x)=f_{m^{\prime}, n^{\prime}} \cdot T^{\left(n^{\prime}\right)} \cdot f_{n^{\prime}}(x)=f_{m^{\prime}, n^{\prime}} \cdot T^{\left(n^{\prime}\right)}\left(x^{\left(n^{\prime}\right)}\right), \\
& \text { for } x^{\left(n^{\prime}\right)} \in \Omega_{n^{\prime}}^{\circ} .
\end{aligned}
$$

Hence, $\left\{T^{\left(n^{\prime}\right)}\right\}$ is consistent and it determines $T$ itself.

Define

$$
\varphi_{k}^{\left(n^{\prime}\right)}(x)=k \text {-th coordinate of } p_{n^{\prime}}(x), x \in S_{\infty},
$$

and denote by $\mathbf{M}_{n^{\prime}}$ a linear manifold of $\mathbf{L}^{2}\left(S_{\infty}\right)$ spanned by $\left\{\varphi_{1}^{\left(n^{\prime}\right)}, \ldots, \varphi_{n^{\prime}}^{\left(n^{\prime}\right)}\right\}$. Since $S_{n^{\prime}}$ is isomorphic to the factor space $S_{\infty} / \eta_{n^{\prime}}$, we can consider $\varphi_{k}^{\left(n^{\prime}\right)}$ as a function of $x^{\left(n^{\prime}\right)} \in S_{n}$. Now we prove

Lemma 3.1. $\left\{\varphi_{1}^{\left(n^{\prime}\right)}, \ldots, \varphi_{n^{\prime}}^{\left(n^{\prime}\right)}\right\}$ is an orthonormal base in $\mathbf{M}_{n^{\prime}}$.

Proof. Let $\left\{\theta_{1}, \ldots, \theta_{n^{\prime}-1}\right\}$ be the polar coordinates of $x^{\left(n^{\prime}\right)} \in S_{n^{\prime}}$. Then they are independent random variables on $\left(S_{n^{\prime}}, \mathbf{B}\left(S_{n^{\prime}}\right), P_{n^{\prime}}\right)$ with joint distribution $\prod_{k=1}^{n^{\prime}-1} f_{k}\left(\theta_{k}\right) d \theta_{1} \cdots d \theta_{n^{\prime}-1}$, where $f_{1}\left(\theta_{1}\right)=1 / 2 \pi, f_{k}\left(\theta_{k}\right)=\Gamma(k / 2)[\sqrt{\pi} \Gamma(k-1 / 2)]^{-1}$ $\sin ^{k-2} \theta_{k}(k \geq 2)$. By expressing $\varphi_{k}^{\left(n^{\prime}\right)}\left(x^{\left(n^{\prime}\right)}\right)=x_{k}^{\left(n^{\prime}\right)}$ with their polar coordinates $\theta_{i}$, it is not hard to show that $\left\{\varphi_{k}^{\left(n^{\prime}\right)} ; k=1,2, \ldots, n^{\prime}\right\}$ is an orthonormal base of $\mathbf{M}^{\prime}$.

THEOREM 3.1. Suppose that the condition $(A)$ is satisfied. If $\left\{\dot{T}_{t}\right\}$ is a measurable flow on $S_{\infty}$ and if $T_{t}$ is consistent with $\left\{\zeta_{n^{\prime}}\right\}$ for each $t$. Then there exists a measurable flow $\left\{T_{t}^{\left(n^{\prime}\right)}\right\}$ on $S_{n^{\prime}}$ for each $n^{\prime} \in M^{\prime}$. They form a consistent system and determine the given $T_{t}$ for every $t$. Furthermore, if the unitary group $\left\{U_{t}\right\}^{\circ}$ 
induced by $\left\{T_{t}\right\}$ reduces the linear manifold $\mathbf{M}_{n^{\prime}}$, of $\mathbf{L}^{2}\left(S_{\infty}\right)$, then $\left\{T_{t}^{\left(n^{\prime}\right)}\right\}$ can be derived from a one-parameter subgroup of $S O\left(n^{\prime}\right)$.

Proof. $1^{\circ}$. Define $T_{t}^{\left(n^{\prime}\right)}$ as in (3.5), then $\left\{T_{t}^{\left(n^{\prime}\right)}\right\}$ is the factor flow of $\left\{T_{t}\right\}$ by (3.5) so that the first part of theorem is a consequence of Proposition 3.1.

$2^{\circ}$. Since $U_{t}$ reduces $\mathbf{M}_{n^{\prime}}$, Lemma 3.1 shows that every $U_{t} \varphi_{k}^{\left(n^{\prime}\right)}$ is expressed in the form

$$
U_{t} \varphi_{k}^{\left(n^{\prime}\right)}=\sum_{j=1}^{n^{\prime}} a_{k j} \varphi_{j}^{\left(n^{\prime}\right)}
$$

Let $(.,$.$) and \langle.,$.$\rangle be the inner product in \mathbf{L}^{2}\left(S_{n^{\prime}}\right)$ and $R^{n^{\prime}}$ respectively. On account of Lemma 3.1, we have

$$
\delta_{k l}=\left(\varphi_{k}^{\left(n^{\prime}\right)}, \varphi_{l}^{\left(n^{\prime}\right)}\right)=\left(U_{t} \varphi_{k}^{\left(n^{\prime}\right)}, U_{t} \varphi_{l}^{\left(n^{\prime}\right)}\right)=\left\langle a_{k}, a_{l}\right\rangle
$$

where $a_{k}=\left(a_{k 1}, \ldots, a_{k n^{\prime}}\right)$. Therefore $A=\left(a_{k l}\right)$ is an orthogonal matrix. On the other hand, the above form implies that

[k-th coordinate of $\left.T_{t}^{\left(n^{\prime}\right)} x\right]=U_{t} \varphi_{k}^{\left(n^{\prime}\right)}(x)=\left\langle a_{k}, x\right\rangle$, for $x=\left(x_{1}, \ldots, x_{n^{\prime}}\right) \in S_{n^{\prime}}$, so that $T_{t}^{\left(\eta^{\prime \prime}\right)}=A$ on $S_{n^{\prime}}$. This concludes the proof of the thorem.

\section{References}

[1] S. Bochner, Harmonic Analysis and the Theory of Probability, Uni. of Calf. Press, 1955.

[2] T. Hida, Finite dimensional approximations to White noise and Brownian motion., J. Math. Mech., (to appear).

[3] T. Hida and H. Nomoto, Gaussian Measure on the Projective Limit Space of Spheres., Proc. Japan Acad., 40 (1964), 301-304.

[4] P. Lévy, Problems concrets d'analyse fonctionelle, Gauthier-Villars, 1951.

[5] H. Totoki, Flow and Entropy, Seminar on Prob., 20 (1964), 1-130 (Japanese).

Nagoya University 